\title{
Visualization of Two-Dimensional Flows of an Ideal Fluid by Computer Technology
}

\author{
Elena $V$. Amosova ${ }^{1 *}$ and Galina P. Ozerova ${ }^{1}$ \\ ${ }^{1}$ France Far Eastern Federal University, Sukhanova str., 8, Vladivostok 690090, Russia
}

\begin{abstract}
The use of application software packages in courses on continuum mechanics is considered as part of the general concept of the use of computer technology in the educational process of engineering specialties at a university. Digital modeling allows you to get an idea of the numerical solution of complex problems of mathematical physics. The powerful capabilities of application programs, combined with a mathematical tool for numerical modeling and graphic post-processing, reproduce the functional relationships between various elements of knowledge. Graphic modeling allows the student in a visual form and in conditions similar to a laboratory experiment to get acquainted with the phenomenon being studied and independently conduct a functional comparison with the corresponding theoretical principles.
\end{abstract}

\section{Introduction}

At the present stage of development of society, it became necessary to use digital technologies in the educational process [1]. The use of a graphic resource in the classroom provides: maximum visibility of the material, improving the quality of knowledge, efficient use of class time, increases the level of cognitive activity of the student $[2,3]$.

Modern e-lerning technologies open up new opportunities for increasing the effectiveness of the educational process in higher education. An important factor in this direction is the formation of students' ability to learn using these technologies even after completing their studies at the university.

One of the promising areas in higher professional education is the use of a blended learning model. The concept of blended learning suggests that in modern conditions it is possible to optimally combine the "strengths" of traditional learning with the advantages of distance learning $[4,5]$. Thus, there is a real opportunity to conduct more interesting and intense full-time classes. The development of information technology allows us to develop a set of computer testing of students. Computer testing accelerates and facilitates the control of students' knowledge, facilitates the performance of classroom tests, is an objective form of knowledge assessment [1,2,3].

* Corresponding author: amosova.ev@dvfu.ru 


\section{Digital modeling}

Digital modeling is used in the study of mathematical models and is reduced to their digital implementation for various numerical input data. The results of these implementations are displayed in graphical or tabular forms. On the examples of problems on the motion of an incompressible fluid $[6,7,8,9,10,11,12]$, the possibilities of using the Gnuplot program [13] for conducting a computational experiment, the purpose of which is to visualize the phenomenon under study, analyze and compare the connections between knowledge elements, are examined [14]. The high graphical interface of the Gnuplot program [15, 16, $17,18,19,20,21,22,23]$ and great opportunities for optimizing the system for solving various scientific problems make it easy to obtain three-dimensional surfaces of potentials of flow velocities, trajectories of flow movements, current lines, diagrams of the distribution of the pressure coefficient on the surface of a streamlined body, etc.

The use of only theoretical foundations in the study of continuum mechanics courses causes difficulty in the perception of this material . A wide graphical representation of twodimensional flows of ideal fluid will provide students with deeper knowledge in the field of fluid and gas mechanics and thereby create the basis for mastering the majors in the specialty. Digital technologies allow students to conduct their own scientific research, to make the work more interesting and reliable.

\subsection{Swirl flow}

Consider the potential motion of an ideal fluid on a plane defined by a complex functional [4]:

$$
W(z)=\frac{Q-\Gamma i}{2 \pi} \ln (z), z=x+i y
$$

where the quantity $\mathrm{Q}$ determines the power of the source, $\Gamma-$ the circulation of the stream. If we take $\Gamma=0, Q \neq 0$, then we get the source potential, in the case of $Q=0, \Gamma \neq 0$ we get the vortex potential. The potential, which is the sum of the source and the vortex, describes the motion of the vortex flow.

Let us construct the surfaces of the velocity potentials describing the fluid motion in the case of the presence of a source (Fig. 1, left) and in the case of a vortex flow (Fig. 1, right). The potential function $\varphi(x, y)$ is determined by the equalities[6]:

$$
\frac{\partial \varphi}{\partial x}=u, \quad \frac{\partial \varphi}{\partial y}=v
$$

where $\vec{v}=\{u(x, y), v(x, y)\}$ is the flow velocity vector. From the continuity equation $\operatorname{div} \vec{v}=0$, we find

$$
\frac{\partial^{2} \varphi}{\partial x^{2}}+\frac{\partial^{2} \varphi}{\partial y^{2}}=0
$$



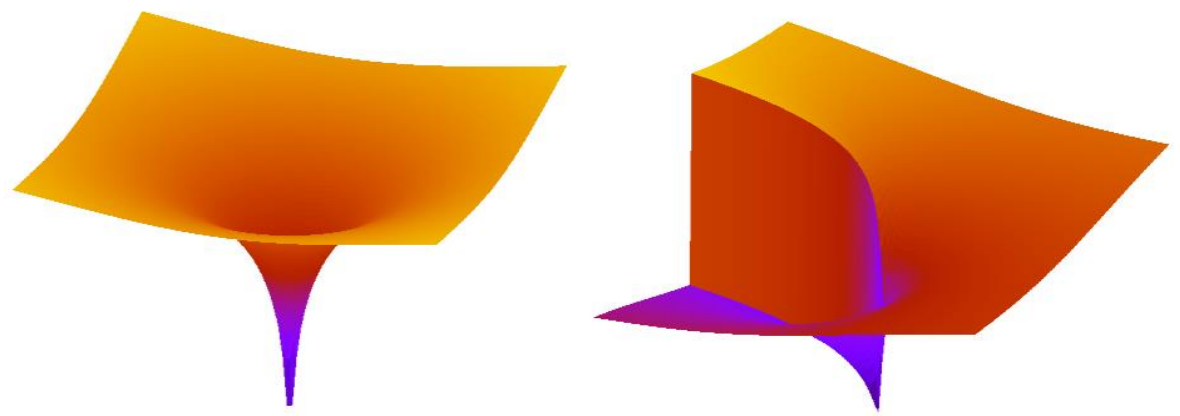

Fig. 1. The potential velocity of a plane flow of an ideal fluid, which describes the motion of the source (left) and vortex flow (right).

The velocity fields of the corresponding flows are shown in Figure 2.
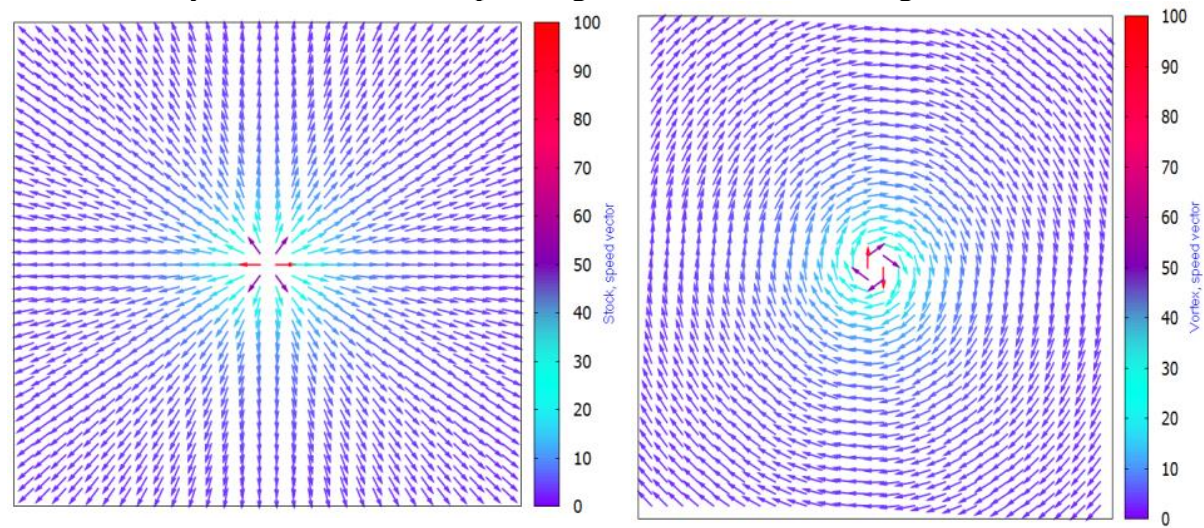

Fig. 2. The velocity field of a plane flow of an ideal fluid, which describes the motion of the source and the vortex flow.

Note that the potential function phi, whose graph is shown in Fig. 1 (left) is the fundamental decision of the Laplace operator on the plane. As is known [12], it is the fundamental solution of the differential operator that belongs to the class of generalized functions.

\subsection{Irrotational flow around a round cylinder}

Consider the complex potential, which is the sum of the complex potentials of a planeparallel flow and dipole:

$$
W(z)=V_{\infty} z+\frac{\mathrm{m}}{2 \pi} \cdot \frac{1}{z}, \quad z=x+i y .
$$

Here $V_{\infty}$ is the free-stream velocity, $\mathrm{m}$ is the dipole moment.

For this flow, the zero streamline is divided into two equations [6]. The first equation is the abscissa axis, a triple circle of radius $a=\sqrt{\left(m / 2 \pi V_{\infty}\right)}$ with center at the origin. On the surface of the cylinder (Fig. 3), the flow velocity takes its maximum value at points with 
coordinates $(0, a),(0,-a)$. Points at which the velocity is zero are called critical, these are points $A(-a, 0), B(a, 0)$.

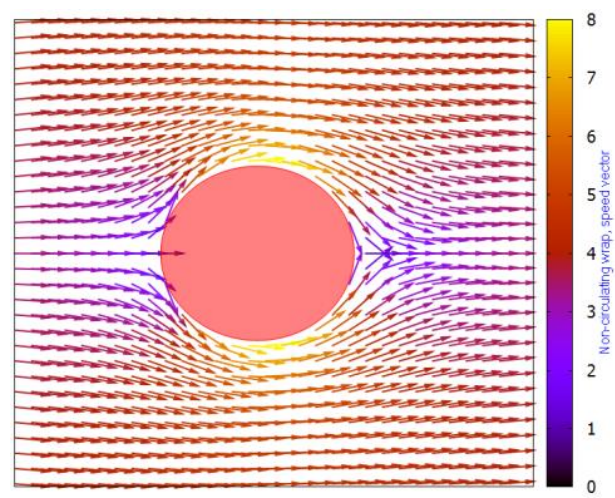

Fig. 3. Velocity field of a flat irrotational flow of an ideal fluid, when flowing around a round cylinder.

From the Bernoulli equation written for the zero streamline, one can find the pressure distribution on the surface of a round cylinder (Fig. 4).

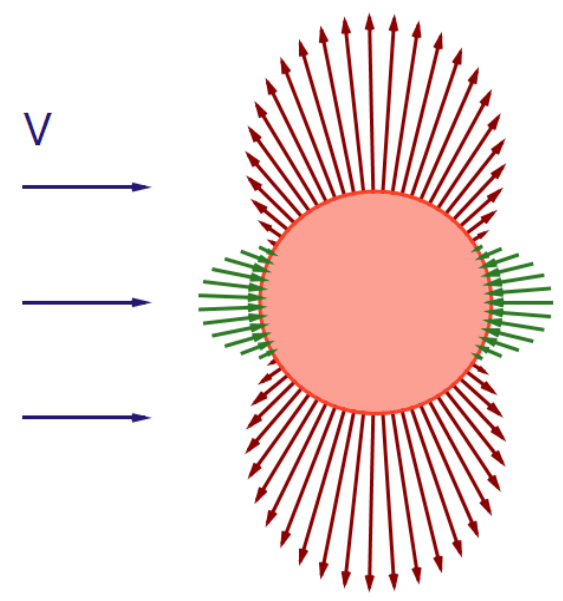

Fig. 4. Pressure coefficient distribution of an irrigation flow of an ideal fluid.

The obtained distribution of dimensionless pressure along the contour of the circle, as follows from (Fig. 4), does not give the resulting force.

Circular flow around a round cylinder

You are free to use colour illustrations for the online version of the proceedings, but any print version will be printed in black and white unless special arrangements have been made with the conference organiser. Please check whether or not this is the case. If the print version will be black and white only, you should check your figure captions carefully and remove any reference to colour in the illustration and text. In addition, some colour figures will degrade or suffer loss of information when converted to black and white, and this should be considered when preparing them. 


\subsection{Circular flow around a round cylinder}

The circulation flow around a circular cylinder can be obtained by applying a vortex with circulation $\Gamma$ to the irrotational flow around a circular cylinder

$$
W(z)=V_{\infty}\left(z+\frac{a^{2}}{z}\right)+\frac{\Gamma i}{2 \pi} \cdot \ln (\mathrm{z}), \quad z=x+i y
$$

Different types of flow are possible depending on the value of circulation $\Gamma$. Using the gnuplot program, we perform a graphical analysis of two types of flows, for $\Gamma<4 \pi a V_{\infty}$ and for $\Gamma>4 \pi a V_{\infty}$. For this, we construct vector fields, draw streamlines, and plot the distribution of the pressure coefficient on the surface of the cylinder.

It can be seen from (Fig. 5, 6) that the smallest velocity value is achieved under the cylinder, which is consistent with the current line containing critical points $A$ and $B$.

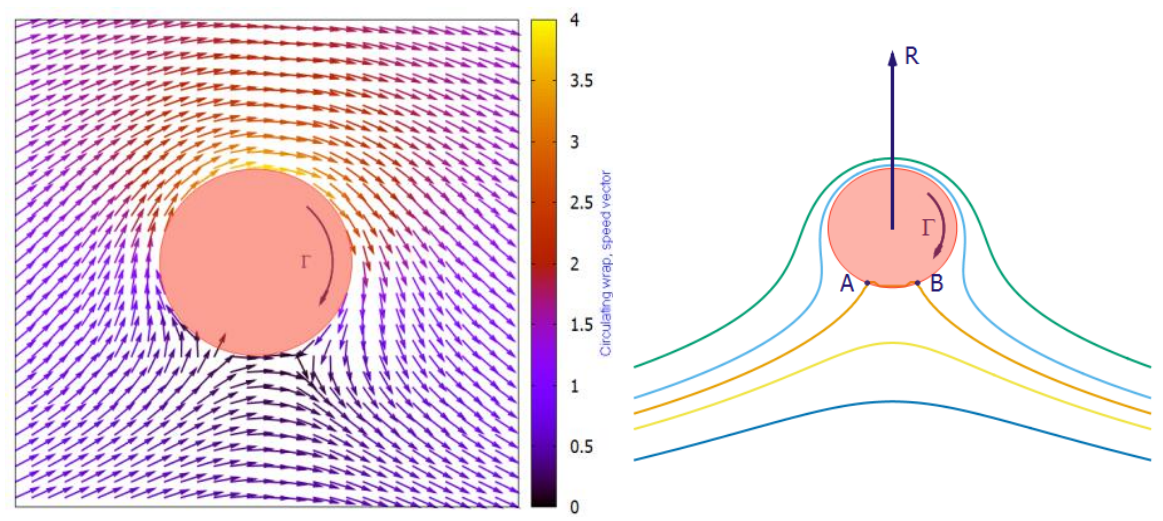

Fig. 5. The velocity field of a plane flow of an ideal fluid (left) and a streamline (right) in the case of a circulation flow around a circular cylinder at $\Gamma<4 \pi a V_{\infty}$.

As in the case of a circulation-free flow around the cylinder, there is no resistance during the circulation flow around, but a transverse force $R$ arises (Fig. 5). With an increase in circulation $\Gamma$, the critical points $A$ and $B$ located on the circle (Fig. 5) will tend to occupy a position inside and outside the circle (Fig. 6), and the magnitude of the lifting force, the vector $R$, will increase. Above the cylinder, the velocity vector takes its maximum value.

Fig. 5, 6 can be presented as an illustration of the definition of a streamline, a line at any point of which the velocity vector is tangential at a given time. This is clearly seen in the case of a streamline containing critical points A and B (Fig. 5, right), (Fig. 6, right). The velocity vectors corresponding to this streamline are colored black $(\mathrm{v}=0)$, (Fig. 5, left), (Fig. 6, left). 

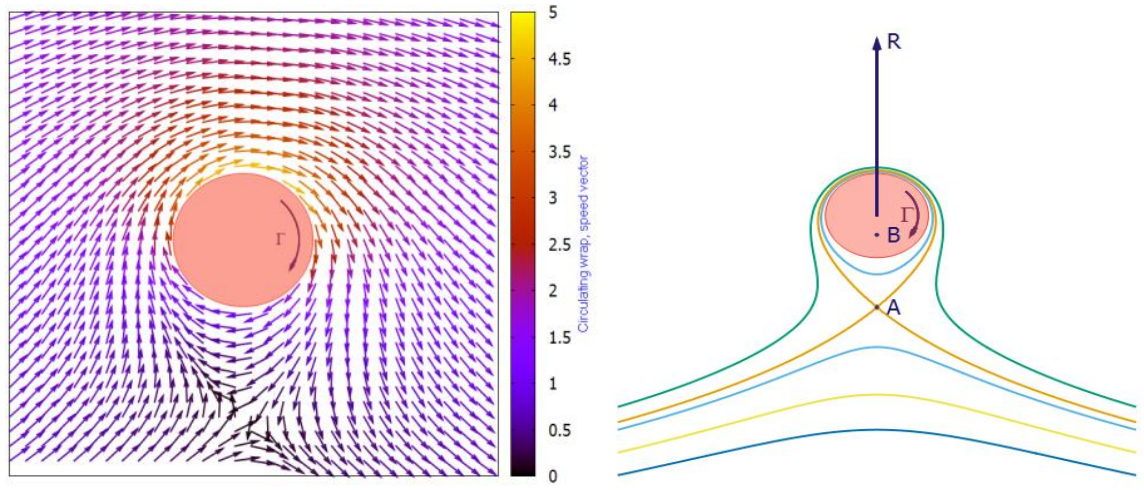

Fig. 6. The velocity field of a plane flow of an ideal fluid (left) and a streamline (right) in the case of a circulation flow around a circular cylinder at $\Gamma>4 \pi a V_{\infty}$.

It can be seen from (Fig. 5, 6) that the lifting force arises due to a change in velocities around the cylinder and, consequently, a change in pressure [6]. Moreover, according to the distribution of the diagrams in (Fig. 7), the pressure above the cylinder decreases to a greater extent than it increases under the cylinder.
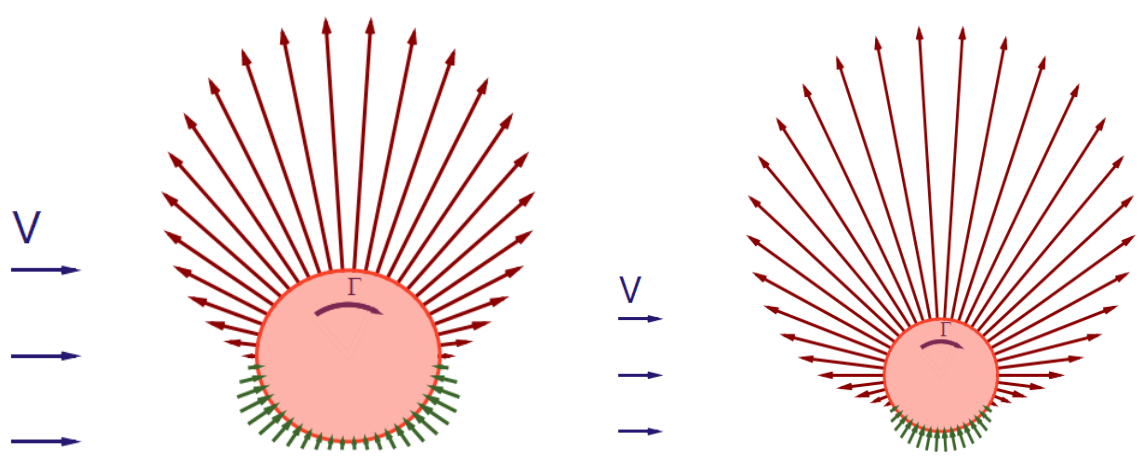

Fig. 7. Distribution of the pressure coefficient on the surface of the cylinder in the case of a circulation flow around a round cylinder, for $\Gamma<4 \pi a V_{\infty}$. (left), for $\Gamma>4 \pi a V_{\infty}$. (right).

\subsection{Fluid flow around an elliptical cylinder}

The complex potential that describes the fluid flow around an elliptical cylinder is given by the formula:

$$
W(z)=\frac{1}{2} \bar{V}_{\infty}\left(\zeta+\frac{V_{\infty}(a+b)^{2}}{c^{2} \cdot \zeta}\right)+\frac{\Gamma i}{2 \pi} \cdot \ln (\zeta), \zeta=z+\sqrt{z^{2}-c^{2}}, z=x+i y .
$$

We determine the incident flux using the complex velocity vector $V_{\infty}=u_{\infty}+i v_{\infty}$, which forms the angle $\Theta_{\infty}$ with the axis $O x$. Let $a$ and $b$ denote the major and minor axis of the ellipse, $c^{2}=a^{2}-b^{2}, \bar{V}_{\infty}=u_{\infty}-i v_{\infty}$. 
Figure 8 shows the velocity field of the irrotational flow around an elliptical cylinder with an ideal fluid flow. The fluid flow is directed at an angle of $45^{\circ}$ to the axis $O x$. The distribution of the pressure coefficient on the cylinder surface corresponding to a given flow is plotted in (Fig. 9).

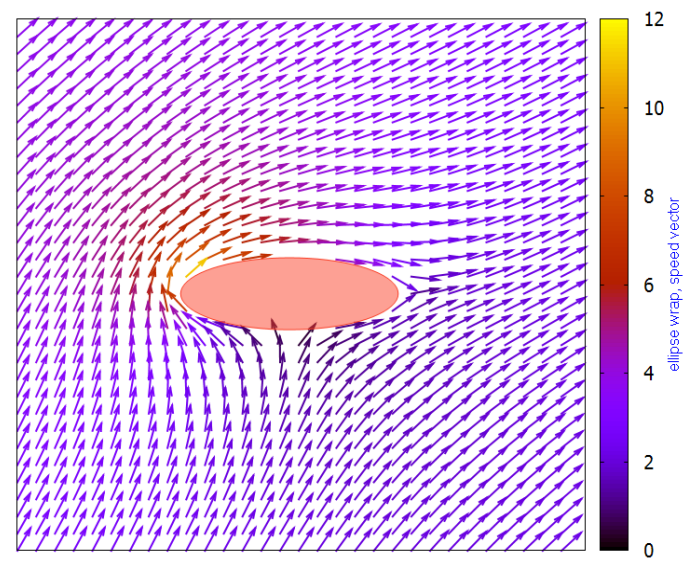

Fig. 8. Velocity field when an ideal fluid flows around an elliptical cylinder, $\Gamma=0$.

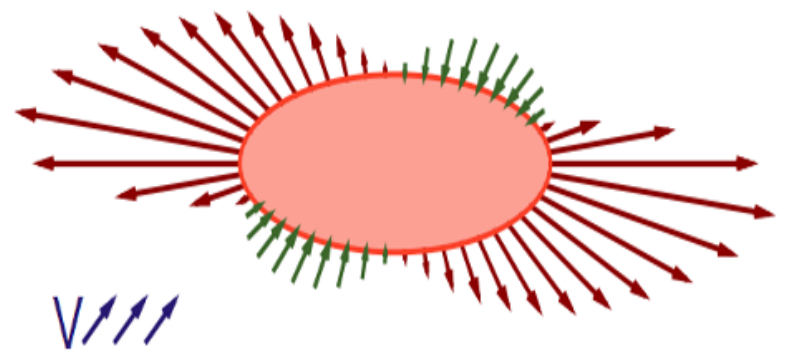

Fig. 9. The distribution of the pressure coefficient on the surface of an elliptical cylinder, $\Gamma=0$.

As in the case of a round cylinder with a irrotational flow, the resulting lift is zero here.

The dynamic effect of the flow on the profile located in it will change if you create a forced vortex around the profile.

Consider the circulation flow around an elliptical cylinder with a given circulation. The velocity field when an ellipse flows around an ideal fluid in the case of positive circulation is shown in (Fig. 10). The incident flow is set at an angle of $45^{\circ}$ to the $O x$ axis. It can be seen from Fig. 10 that a circulation flow forms around the cylinder, carrying the critical point down and to the right. 


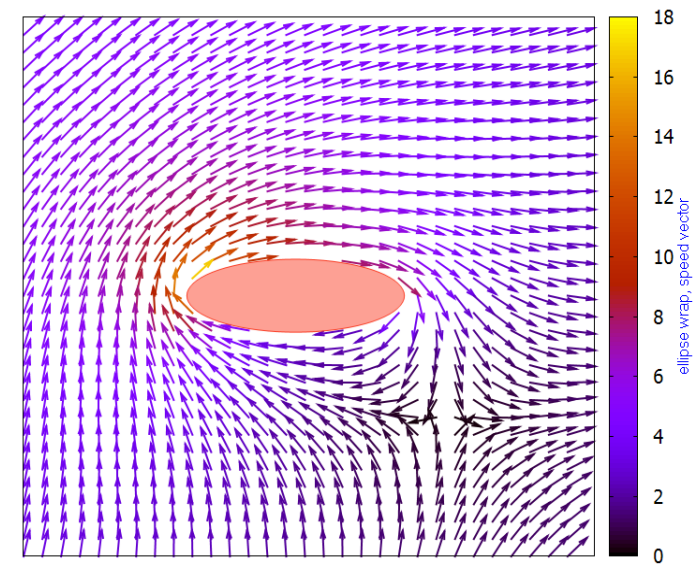

Fig. 10. The velocity field in the circulation flow around an elliptical cylinder.

As in the case of a circulation-free flow around the cylinder, there is no resistance in the circulation flow around, but a transverse force arises equal to the product of the fluid density and the flow velocity and the circulation $\vec{R}=\rho \Gamma \vec{V}_{\infty}$.

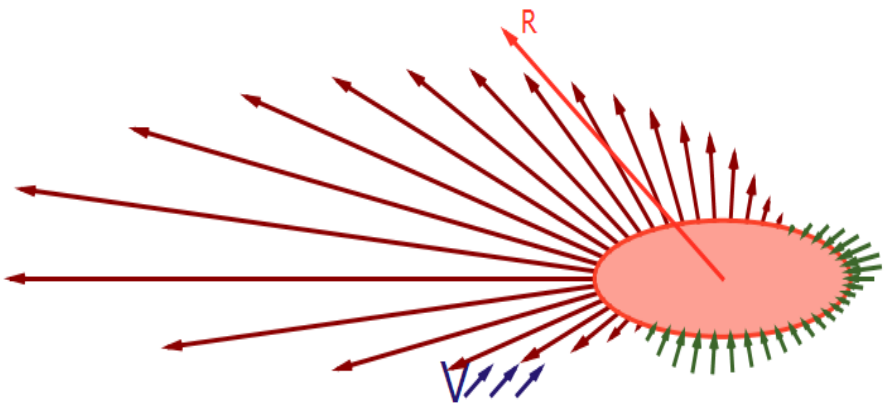

Fig. 11. Distribution of the pressure coefficient on the profile surface during the circulation flow around an elliptical cylinder.

We note that the only force acting on the profile in an irrotational flow of an ideal fluid is a force perpendicular to the direction of the flow, which can be called the lifting force. This is a special case of the d'Alembert paradox $[6,7,8]$.

Consider the flow around a plate with a smooth exit of jets from the trailing edge. We write the complex potential of the flow around the plate:

$$
W(z)=u_{\infty} z-i v_{\infty} \sqrt{z^{2}-c^{2}}+\Gamma i c v_{\infty} \cdot \ln \left(z+\sqrt{z^{2}-c^{2}}\right), \quad z=x+i y
$$



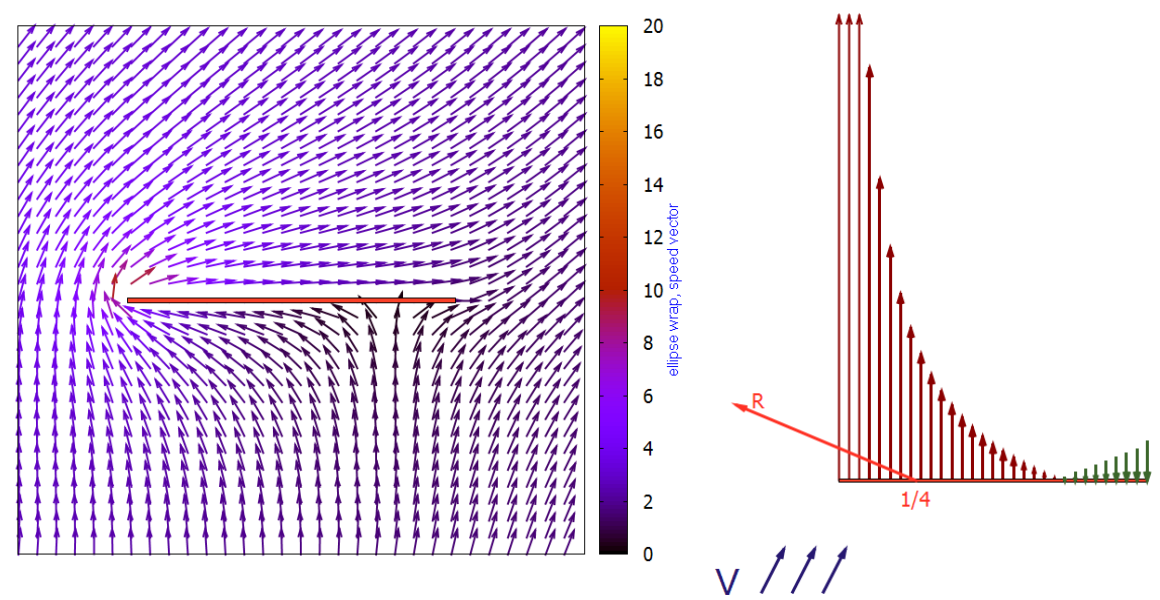

Fig. 12. Velocity field and distribution of the pressure coefficient of the fluid flow around the plate.

The center of pressure of the flow on the plate is raised a quarter of its length from the leading edge, and the position of the center of pressure does not depend either on the flow velocity or on the angle of attack.

During the rotational motion of bodies in a real fluid with internal friction (viscosity), one can observe the occurrence of circulating motions, qualitatively similar to those just studied. The effect of the formation of transverse forces (Magnus effect) helps to explain many interesting phenomena. Such, for example, is the appearance of the aerodynamic moment of the action of the air flow on a rotating artillery shell, which, together with the gyroscopic moment, leads to the rotation of the shell in the plane of fire and its axis approaching the tangent to the trajectory.

\section{Conclusion}

In connection with the development of computer technology and the emergence of powerful mathematical software packages, it became possible to quickly build and test mathematical models of varying complexity. In this case, the most interesting are the simplest models that give satisfactory results for practice in terms of adequacy. To date, there is no such computer program or technology that can not only simplify the calculation of a flowing fluid, but completely solve this problem, which will greatly facilitate the work on finding a solution. The paper describes a technique for studying the motion of homogeneous flows using the example of an ideal fluid using computer technology. A comparison is made of the values of the pressure function on the surface of a symmetric body with a flow around a flow with a given circulation and without circulation. A demonstration of the d'Alembert paradox is given. The visualization of the characteristics of the incoming flow by means of computer technologies is presented.

The various flows of an ideal fluid presented in the work can be used as a demonstration material when studying the course "mechanics of fluid and gas".

\section{References}

1. R.M. Timerbaev \& V.Y. Shurygin, Pedagogic Condition and Methodological Aspects of Education Intensification on the Course "Theoretical Mechanics», in Life Science Journal, 12(12), 405-408 (2014) 
2. D.M. Fraser, R. Pillay, L. Tjatindi \& J.M. Case, Enhancing the learning of fluid mechanics using computer simulations, in Journal of Engineering Education, 96(4), 381-388 (2014)

3. F. Bertacchini, E. Bilotta, F. Caldarola\& P. Pantano, The role of computer simulations in learning analytic mechanics towards chaos theory: a course experimentation, in International Journal of Mathematical Education in Science and Technology, 50(1), 100-120, (2019)

4. G.P. Ozerova, G.F. Pavlenko, Prediction of student performance in blended learning by utilizing learning analytics data, in Science for Education Today, 6 (2019)

5. G. Ozerova, P. Lobodin, Ispol'zovaniye modeli smeshannogo obucheniya dlya organizatsii uchebnogo protsessa v vuze, in Otkrytoye i distantsionnoye obrazovaniye, 3(63), pp. 11-19 (2016)

6. L.G. Loytsyanskiy, Mekhanika zhidkostey i gazov, Drofa, Moscow, 840 (2003)

7. L.I. Sedov, C.K. Chu, H. Cohen, B. Seckler \& J. Gillis, Two-dimensional problems in hydrodynamics and aerodynamics, in Physics Today, 18, 62 (1965)

8. L.D. Landau \& E.M. Lifshitz, Fluid mechanics (1987)

9. N.E. Kochin, I.A. Kibel' \& N.V. Roze, Theoretical hydromechanics, in Interscience publishers (1964).

10. A.J. Chorin, J.E. Marsden \& J.E. Marsden, A mathematical introduction to fluid mechanics (Vol. 3), New York: Springer (1990).

11. B.R. Munson, T.H. Okiishi, W.W. Huebsch \& A.P. Rothmayer, Fluid mechanics. Singapore: Wiley (2013)

12. S.G.L. Smith, How do singularities move in potential flow?, in Physica D: Nonlinear Phenomena, 240(20), pp. 1644-1651 (2011)

13. S.M. Ramodanov, Motion of a circular cylinder and a vortex in an ideal fluid, in Regular and chaotic dynamics, 6(1), pp. 33-38, (2001)

14. P.K. Janert, Gnuplot in action: understanding data with graphs, Manning, (2010)

15. J. Bernhard, Teaching engineering mechanics courses using active engagement methods, in Physics Teaching in Engineering Education (PTEE 2000), 13-17 June 2000, Budapest, Hungary (2000)

16. N.M. O’Boyle, Book Review of Gnuplot in Action, (2008)

17. T. Williams, C. Kelley, R. Lang, D. Kotz \& J. Campbell, 1 gnuplot (2008)

18. J. Racine, gnuplot 4.0: a portable interactive plotting utility, in Journal of Applied Econometrics, 21(1), pp. 133-141 (2006)

19. B.G. Moore, Orbital plots using gnuplot, in Journal of Chemical Education, 77(6), p. 785 (2000)

20. T. Williams, C. Kelley, H.B. Bröker, J. Campbell, R. Cunningham, D. Denholm \& L. Hecking, gnuplot 4.6. An Interactive Plotting Program (2012)

21. A. Liaw, D. Crawford \& R. D. A. Logicon, Gnuplot 3.5* user's guide. na, (1995)

22. T. Williams \& C. Kelley, GNUplot: an interactive plotting program (1998)

23. L. Phillips, gnuplot Cookbook, in Packt Publishing Ltd (2012) 